\title{
Adaptive changes in supporting cells of olfactory epithelium in fishes during their spawning period
}

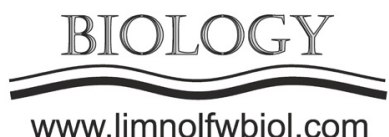

\author{
Klimenkov I.V. ${ }^{1 *}$, Sudakov N.P. ${ }^{1}$, Pastukhov M.V. ${ }^{2}$, Kositsyn N.S. ${ }^{3}$ \\ ${ }^{1}$ Limnological Institute, Siberian Branch, Russian Academy of Sciences, 3 Ulan-Batorskaya St., Irkutsk, 664033, Russia. \\ ${ }^{2}$ Vinogradov Institute of Geochemistry, Siberian Branch, Russian Academy of Sciences, 1a Favorsky St., Irkutsk, 664033, Russia. \\ ${ }^{3}$ Institute of Higher Nervous Activity and Neurophysiology, Russian Academy of Sciences, 5a Butlerova St., Moscow, 117485, Russia.
}

\begin{abstract}
This research was devoted to studying the olfactory system adaptation mechanisms in fishes during their spawning period. For this purpose, the morpho-functional changes of supporting cells in the olfactory epithelium were analyzed by electron transmission and laser confocal microscopy. We have indicated that active mitochondria are concentrated in the apical zone of ciliated supporting cells. This position of mitochondria is important for the energy supply of cilia to enhance the olfactory mucus circulation. Moreover, there was an increase in mucous supporting cells secretory activity. Generally, identified changes in olfactory epithelium aim to raise the effectiveness of sex pheromone reception during the spawning period.
\end{abstract}

Keywords: olfactory epithelium, supporting cells, mitochondria, secretion, spawning

The study of the adaptive functioning mechanisms of the olfactory system is one of the important problems of sensory physiology. Cellular chemoreception at the level of the peripheral olfactory analyzer has essential significance in the genesis of these adaptations (Doty, 2015). Nevertheless, clear correlations between structural polymorphism of receptor neurons, their sensitivity, and realization of specific behavior were not registered in animals (Hamdani et al., 2008). Chemical communications between hydrobionts induce various functional changes in all parts of the olfactory system (Harden et al., 2006). This allows animals to identify proper sexual partners and synchronize their germ cells development. In the olfactory epithelium, the receptor neurons are in close neighborhood with supporting cells that are glial cells functional analogs. These cells play an important role in olfactory epithelium activity regulation (Getchell, 1986), metabolism of xenobiotics and odorants (Débat et al., 2007), secretion of regulatory and defensive molecules (Laudien et al., 2011) as well as production and circulation of the olfactory mucus (Bigday and Samoilov, 2015). All these functions are essential for effective odor reception. Thus, the aim of our research to study adaptive rearrangements of olfactory epithelium support cells in fishes during their spawning period.

The comparative study of olfactory rosettes was carried out on endemic fish in Lake Baikal, Cottocomephorus grewingkii Dybowski, 1874. The olfactory epithelium ultrastructure was analyzed by transmission electron microscopy (Leo 906 E, Zeiss), and functional status of mitochondria was studied with laser confocal microscopy (LSM 710, Zeiss) (Klimenkov et al., 2018; 2020).

The analysis of structural and functional peculiarities of olfactory epithelium in spawning fishes has revealed significant adaptive changes in supporting cells. One of the specific differences is an increase in mitochondria count and their redistribution in cells: these organelles are concentrated near the basal bodies in the apical part of ciliated supporting cells. The data on staining with Mitotracker orange CMTMRos (ThermoFisher Scientific) suggest a high level of their energy production. An increase in mitochondria quantity (2.3-fold, $\left.\mathrm{p}_{\mathrm{u}} \leq 0.05\right)$ indicates a high energy consumption by cilia for intensification of their movement. This may increase the effectiveness of pheromone action at extremely low concentrations (Walaszczyk et al., 2013). The total number of mitochondria in the epithelium during the spawning period is higher than before spawning. This characterizes the general increase in the energy metabolism of the fish olfactory epithelium, which is very important for the reception of essential signals for reproduction.

Another important feature of supporting cells at this stage of ontogenesis is a significant increase in secretory activity of mucous cells. In the cytoplasm of these cells, we observed the uniform extension of rough endoplasmic reticulum with high ribosome number. All cisternae of the Golgi apparatus also undergo the

*Corresponding author.

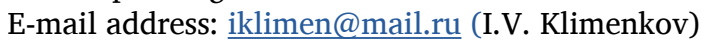

(C) Author(s) 2020. This work is distributed under the Creative Commons Attribution 4.0 License. 
extension. Near the Golgi complex, numerous vacuoles of various sizes appear. We may assume that this process changes the mucus properties that can affect the odorant reception.

Interestingly, the interpenetration areas exist between ciliated and secretory supporting cells. These forms of intercellular contacts are registered in epithelium as before and during spawning. Moreover, cytoplasmic sprouts of secretory cell penetrating into the ciliated cell carry secretory vesicles. We assume that this sprouts containing secretory vesicles can be captured with ciliated cells by endocytosis. This process can be essential for exchange by nutrients and signals between these cells.

On the whole, during the spawning period, the supporting cells of olfactory epithelium in fishes undergo significant morpho-functional changes. The further study of these basic processes is important for the development of neurobiology, medicine, and other scientific fields related to the research of human and animal behavior.

This study was performed in the Electron Microscopy Center of collective instrumental center "Ultramicroanalysis" Limnological Institute of the Siberian Branch of the Russian Academy of Sciences (http://www.lin.irk.ru/copp/rus/) and supported by the Russian Foundation for Basic Research, Project Nos. 18-04-00816a, and 19-05-50017.

\section{References}

Bigday E.V., Samoilov V.O. 2015. Chemotaxis as a mechanism of locomotor activity of olfactory cilia. Biophysics 60: 588-591. DOI: 10.1134/S0006350915040041
Débat H., Eloit C., Blon F. et al. 2007. Identification of human olfactory cleft mucus proteins using proteomic analysis. Journal of Proteome Research 6(5): 1985-1996. DOI: $10.1021 /$ pr0606575

Doty R.L. 2015. Olfactory dysfunction and its measurement in the clinic. World Journal of Otorhinolaryngology - Head and Neck Surgery 1(1): 28-33. DOI: 10.1016/j.wjorl.2015.09.007

Getchell T.V. 1986. Functional properties of vertebrate olfactory receptor neurons. Physiological Reviews 66(3): 772-818. DOI: 10.1152/physrev.1986.66.3.772

Hamdani el H., Lastein S., Gregersen F. et al. 2008. Seasonal variations in olfactory sensory neurons--fish sensitivity to sex pheromones explained? Chemical Senses 33(2): 119-123. DOI: 10.1093/chemse/bjm072

Harden M.V., Newton L.A., Lloyd R.C. et al. 2006. Olfactory imprinting is correlated with changes in gene expression in the olfactory epithelia of the zebrafish. Journal of Neurobiology 66(13): 1452-1466. DOI: 10.1002/neu.20328

Klimenkov I.V., Sudakov N.P., Pastukhov M.V. et al. 2018. Rearrangement of actin microfilaments in the development of olfactory receptor cells in fish. Scientific Reports 8(1). DOI: 10.1038/s41598-018-22049-7

Klimenkov I.V., Sudakov N.P., Pastukhov M.V. et al. 2020. The phenomenon of compensatory cell proliferation in olfactory epithelium in fish caused by prolonged exposure to natural odorants. Scientific Reports 10(1). DOI: 10.1038/ s41598-020-65854-9

Laudien M., Dressel S., Harder J. et al. 2011. Differential expression pattern of antimicrobial peptides in nasal mucosa and secretion. Rhinology 49(1): 107-111. DOI: 10.4193/ Rhino10.036

Walaszczyk E.J., Johnson N.S., Steibel J.P. et al. 2013. Effects of sex pheromones and sexual maturation on locomotor activity in female sea lamprey (Petromyzon marinus). Journal of Biological Rhythms 28(3): 218-226. DOI: $10.1177 / 0748730413488994$ 